\title{
Sausage and kink oscillations in incompressible annular magnetic cylinders
}

\author{
B. K. Carter and R. Erdélyi
}

Solar Physics \& Space Plasma Research Centre (SP $\left.{ }^{2} \mathrm{RC}\right)$, Dept. of Applied Maths, The University of Sheffield, Hicks Building, Hounsfield Road, Sheffield, S3 7RH, UK

e-mail: [robertus; b.carter]@sheffield.ac.uk

Received 24 January 2007 / Accepted 30 July 2007

\begin{abstract}
Aims. The propagation of MHD waves in a structured magnetic flux tube embedded within a straight magnetic environment is studied analytically. The motivation for this analysis comes from the observations of damped loop oscillations indicating the possibility that only part of the loop is homogeneous in the radial direction and from the observation of two simultaneous waves with different speeds in the same magnetic loop in the solar atmosphere.

Methods. The general dispersion relation of longitudinal wave propagation is derived for a flux tube configuration consisting of a core, annulus and external region each with straight distinct magnetic field. Modes of oscillation are examined from the general dispersion relation that is suitable for obtaining information not just on oscillations but also on some instability properties of this complex tube structure. Specific attention is given to the modification of the phase speeds and oscillation periods caused by the annulus structure, compared to a single monolithic tube.

Results. It is shown that two purely surface modes arise. The relative change in periods due to the additional annulus layer is shown to be rather significant (up to $20 \%$ in some cases). In particular we found that in photospheric type annular tubes, periods are less than their counterparts in a monolithic loop while for a dense flux tube in the majority of cases the period was greater than the period of the counterpart monolithic flux tube. Both short and long wavelength approximations are considered for both the symmetrical (sausage) and kink modes.

Conclusions. Annular structuring of a magnetic flux tube will reduce or increase periods of the allowed oscillations depending on the type of flux tube considered (either as a magnetic or as a density enhancement). The results are relevant to further our knowledge of solar magneto-seismology. In particular, the obtained results for the kink oscillations may be applicable from photosphere to corona, while the case for the sausage mode is more relevant in the lower part of the solar atmosphere.
\end{abstract}

Key words. magnetohydrodynamics (MHD) - waves - Sun: oscillations - Sun: magnetic fields

\section{Introduction}

The solar atmosphere, from the photosphere to the corona, has an array of magnetically structured features and recent observation equipment (e.g. SOlar and Heliospheric Observatory (SOHO)/EIT, Transition Region And Coronal Explorer (TRACE)) and advanced techniques have led to an explosion of the number of theoretical ideas (for an extensive book review on observations see Aschwanden 2004) initiated by the discovery of periodic disturbances of various solar structures. For a review of MHD oscillations see e.g. Roberts (2000); Aschwanden (2003); Nakariakov \& Roberts (2003); Wang (2004); Nakariakov \& Verwichte (2005); Banerjee et al. (2007).

Many different new theoretical models have been spurred on by observations that started with transverse (kink) standing oscillations of fast magnetosonic waves (Aschwanden et al. 1999; Nakariakov et al. 1999). Propagating kink modes have also been observed along with derived measurements of amplitudes, periods, damping times and magnetic tube parameters (e.g. Kukhianidze et al. 2006). Fluctuations in sunspot umbrae and penumbrae have been observed with periodicities of 3 , and 5 min being prominent (for a review see e.g. Bogdan 2000). Running penumbral waves, the dominant oscillating phenomena in the sunspot penumbra, are one among the vast array of photospheric/chromospheric observations (see e.g. Christopoulou et al. 2000, 2001; Georgakilas et al. 2000, 2002; Kobanov 2000). These wave disturbances commonly display a disc centre-to-limb asymmetry across the sunspot suggesting that the oscillatory motions are aligned with the penumbral magnetic field (see review by Bogdan \& Judge 2006). It is possible that these running waves are travelling in magnetic tubes, or photospheric fibrils.

Solar magneto-seismology is a current subject that has evolved from the eruption of wave and oscillation observations in the entire magnetised solar atmosphere (see Roberts et al. 1984). It has recently taken a direction of analysing the direct coupling between the solar interior and atmosphere (e.g. De Pontieu et al. 2003, 2004, 2005; Erdélyi 2006) and the exploration into inferring diagnostic information about solar magnetic waveguides using the two-dimensional nature of the available high-resolution imaging observations (e.g. Erdélyi \& Verth 2007; Verth et al. 2007). However, more detailed elaborate theory and improved observations are required to develop the method of seismology applicable in the structured solar atmosphere. It was found that the existence of inhomogeneities in the form of structuring of the magnetic field enables flux tubes, or fibrils, to act as wave guides for a variety of modes. The typical speeds arising are linked closely to the local Alfvén speed $v_{\mathrm{A}}$, sound speed $c_{\mathrm{S}}$ and tube speed $c_{\mathrm{T}}$. Sub-resolution flux 
tube structure is still more a matter of some speculation. As a specific example; combined data of May 131998 from both the EIT instrument on SOHO and from TRACE show the simultaneous observation of two slow magnetosonic waves propagating along a perceived coronal loop with speeds of 95 and $110 \mathrm{~km} \mathrm{~s}^{-1}$ (Robbrecht et al. 2001). This observation was interpreted by the authors as temperature differences within the observed loop hinting at a substructure of perhaps either concentric shells of different temperatures or of thin strands within the same loop at different temperatures. There is no conclusive proof disputing these possible flux tube structures nor preference given towards one in particular.

Here we shall assume that a flux tube consists of a central core surrounded by a shell, or annulus, layer. It is of interest to understand the role of this annulus layer upon the phase speed and mode existence of waves supported by such a system. Mikhalyaev \& Solov'ev (2005); Erdélyi \& Carter (2006) used the geometric model of a core and shell structured flux tube to investigate the propagation of ideal MHD waves and the effect this structuring has on wave speeds and periods. Mikhalyaev \& Solov'ev (2005) studied the structuring of an annular tube with straight magnetic field. Their study led to a dispersion relation describing wave propagation in such a model for a compressible medium and they discuss asymptotic expansion, slow and fast modes for coronal conditions only and damping times of the fast magnetosonic wave. Erdélyi \& Carter (2006), for an incompressible medium, studied analytically linear sausage oscillations in a tube consisting of a core and magnetically twisted annulus in detail. Here, however, we consider solely a straight axial configuration but extend the study to both sausage and kink modes, and, specifically to the effect the annulus layer has on the MHD wave modes found. The assumption of incompressibility reduces the direct full applicability to coronal loops except for kink modes as they are highly incompressible perturbations (see e.g. Edwin \& Roberts 1983; Ryutov \& Ryutova 1976). However, the model is more applicable to lower atmospheric oscillations (e.g. from sub-photospheric tubes to chromospheric fibrils). In this study we model the flux tube with inhomogeneous layer as a straight magnetic core surrounded by a straight magnetic annulus embedded in a straight ambient magnetic environment. For simplicity we neglect the effects of compressibility and of gravity, the emphasis being on the role of the magnetic field and the annulus layer on existing modes. We find the general dispersion relation for degenerated magnetosonic waves (of course, in incompressible plasma, the sound speed is infinite and appropriate limits must be taken as it will be shown in the paper). The general dispersion relation is solved analytically for the sausage and kink cases and also in the long wavelength (thin tube) and short wavelength (thick tube) approximations. We find two surface modes (in the incompressible approximation, for a straight magnetic field, body modes are removed from the system) occurring at the two natural surfaces of the system. The modifications to the phase speeds are shown to be significant and dependent not only on the Alfvén speed in each region but also on the ratio $a / R$ of the core and annulus radii.

\section{The magnetic annulus}

\subsection{Equilibrium}

We restrict our investigation to an incompressible plasma for which the phase speeds of the slow and Alfvén waves, in the limit of incompressibility, become indistinguishable, the modes are discernable through direction of perturbation only (Alfvén

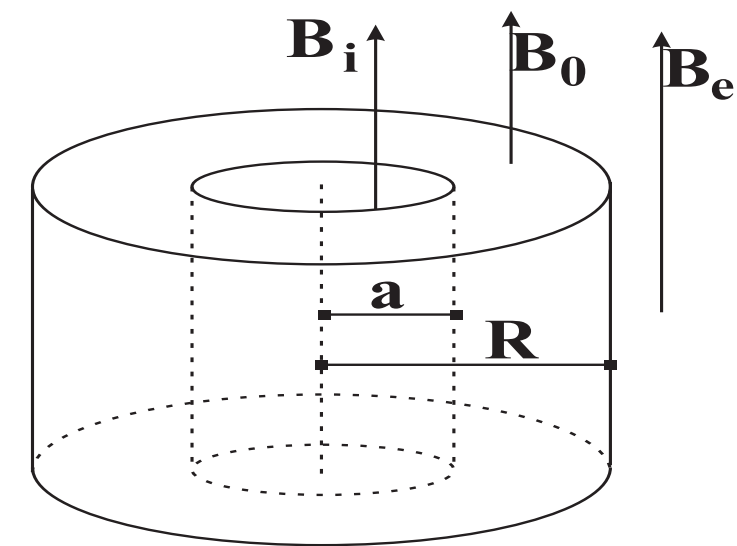

Fig. 1. The equilibrium configuration of a magnetic cylinder consisting of a core, annulus and external regions, all with straight magnetic field.

wave perturbations are perpendicular to both magnetic field and propagation vector while slow wave perturbations are in the same plane). The fast waves are removed from the system. Straight flux tubes, even without gravity, as studied by Edwin \& Roberts (1983, hereafter ER83), have been predicted to be inhomogeneous across their diameter. This inhomogeneity is modelled as an annulus (or shell) layer covering a percentage of the tube's radius as shown in Fig. 1. The modes of oscillation in a straight configuration of magnetic core, annulus and ambient external field are studied here. To do this we consider, in cylindrical coordinates, the equilibrium magnetic field of the form

$\boldsymbol{B}=\left(0,0, B_{z}(r)\right)$

embedded in an incompressible plasma. For the sake of simplicity we take the plasma density $\rho$ as uniform and the plasma pressure $p(r)$ structured in the radial direction as to satisfy the radial pressure balance

$\frac{\mathrm{d} p}{\mathrm{~d} r}+\frac{\mathrm{d}}{\mathrm{d} r}\left(\frac{B_{z}^{2}}{2 \mu}\right)=0$

in which $\mu$ is the magnetic permeability and $B_{z}$ denotes the equilibrium field strength.

\subsection{The $M H D$ equations}

We consider a longitudinal magnetic field in each region of a magnetic annulus so that

$\boldsymbol{B}= \begin{cases}\boldsymbol{B}_{\mathrm{i}}=\left(0,0, B_{\mathrm{i}}\right), & r<a, \\ \boldsymbol{B}_{0}=\left(0,0, B_{0}\right), & a \leq r \leq R, \\ \boldsymbol{B}_{\mathrm{e}}=\left(0,0, B_{\mathrm{e}}\right), & r>R,\end{cases}$

where $B_{\mathrm{i}}, B_{0}, B_{\mathrm{e}}$ are constant. We take the densities in the core, annulus and external regions as $\rho_{\mathrm{i}}, \rho_{0}, \rho_{\mathrm{e}}$, respectively, and similarly denote the pressure in each region as $p_{\mathrm{i}}, p_{0}, p_{\mathrm{e}}$. The pressure balance at the boundaries $r=a$ and $r=R$ (Eq. (2)) gives the relations

$p_{\mathrm{i}}+\frac{B_{\mathrm{i}}^{2}}{2 \mu}=p_{0}+\frac{B_{0}^{2}}{2 \mu}, \quad p_{0}+\frac{B_{0}^{2}}{2 \mu}=p_{\mathrm{e}}+\frac{B_{\mathrm{e}}^{2}}{2 \mu}$,

and we denote $v_{\mathrm{Ai}}=B_{\mathrm{i}} /\left(\mu \rho_{\mathrm{i}}\right)^{1 / 2}, v_{\mathrm{A} 0}=B_{0} /\left(\mu \rho_{0}\right)^{1 / 2}$ and $v_{\mathrm{Ae}}=$ $B_{\mathrm{e}} /\left(\mu \rho_{\mathrm{e}}\right)^{1 / 2}$ as the Alfvén speeds in the internal, annulus and external regions, respectively.

Taking linear perturbations of the ideal MHD equations about this equilibrium and Fourier-decomposing the total 
Lagrangian pressure $p_{\mathrm{T}}(=p+\boldsymbol{B} . \boldsymbol{b} / \mu$ for perturbed field $\boldsymbol{b}$ and plasma pressure $p$ ) and normal component of Lagrangian displacement $\xi_{r}$ like

$\left(p_{\mathrm{T}}, \quad \xi_{r}\right) \sim\left(\hat{p}_{\mathrm{T}}(r), \quad \hat{\xi}_{r}(r)\right) \mathrm{e}^{\mathrm{i}\left(m \theta+k_{z} z-\omega t\right)}$,

and omitting the hat of the Fourier decomposed perturbations for the sake of simplicity of notation, we find that $p_{\mathrm{T}}(r)$ satisfies the Bessel equation

$\frac{\mathrm{d}^{2} p_{\mathrm{T}}}{\mathrm{d} r^{2}}+\frac{1}{r} \frac{\mathrm{d} p_{\mathrm{T}}}{\mathrm{d} r}-\left(k_{z}^{2}+\frac{m^{2}}{r^{2}}\right) p_{\mathrm{T}}=0$

where $m$ is the azimuthal wavenumber (see e.g. Edwin \& Roberts 1983).

\subsection{The dispersion relation}

We combine the magnetic configuration model defined by Eq. (3) with the surface wave solutions for $p_{\mathrm{T}}$ of the Bessel equation, Eq. (6), which are a linear combination of $I_{m}\left(k_{z} r\right)$ and $K_{m}\left(k_{z} r\right)$, the modified Bessel functions, of order $m$. We require that $p_{\mathrm{T}}$ is regular at $r=0$ and the solutions are evanescent as $r \rightarrow \infty$ (i.e. surface waves) giving

$p_{\mathrm{T}}= \begin{cases}\alpha I_{m}\left(k_{z} r\right), & r<a, \\ \beta I_{m}\left(k_{z} r\right)+\gamma K_{m}\left(k_{z} r\right), & a \leq r \leq R, \\ \delta K_{m}\left(k_{z} r\right), & r>R,\end{cases}$

for arbitrary constants $\alpha, \beta, \gamma$ and $\delta$. For the solution and dispersion relation for the configuration of a magnetic annulus we require the solutions for $p_{\mathrm{T}}$ in Eq. (7) to satisfy the continuity of total Lagrangian pressure $p_{\mathrm{T}}$ and the continuity of the normal displacement perturbation, $\xi_{r}$, across the boundaries $r=a$ and $r=R$ :

$\left[\xi_{r}\right]_{r=a+\varepsilon}^{r=a-\varepsilon}=\left[\xi_{r}\right]_{r=R+\varepsilon}^{r=R-\varepsilon}=0, \quad \varepsilon \rightarrow 0$,

$\left[p_{\mathrm{T}}\right]_{r=a+\varepsilon}^{r=a-\varepsilon}=\left[p_{\mathrm{T}}\right]_{r=R+\varepsilon}^{r=R-\varepsilon}=0, \quad \varepsilon \rightarrow 0$.

We recall that (see Erdélyi \& Carter 2006, for details)

$\xi_{r}=\frac{1}{\rho\left(\omega^{2}-\omega_{\mathrm{A}}^{2}\right)} \frac{\mathrm{d} p_{\mathrm{T}}}{\mathrm{d} r}$

and so, from the expression for $p_{\mathrm{T}}$ in each region (given by Eq. (7)), we can, after some algebra, consolidate these to a single equation. This is the dispersion relation for the configuration of a straight magnetic annulus defined by Eq. (3):

$$
\begin{gathered}
\frac{Q_{0}^{\mathrm{i}} K_{m}^{\prime}\left(k_{z} a\right)-\left(I_{m}^{\prime}\left(k_{z} a\right) K_{m}\left(k_{z} a\right) / I_{m}\left(k_{z} a\right)\right)}{I_{m}^{\prime}\left(k_{z} a\right)\left(Q_{0}^{\mathrm{i}}-1\right)}= \\
\frac{K_{m}^{\prime}\left(k_{z} R\right)\left(Q_{0}^{\mathrm{e}}-1\right)}{Q_{0}^{\mathrm{e}} I_{m}^{\prime}\left(k_{z} R\right)-\left(K_{m}^{\prime}\left(k_{z} R\right) I_{m}\left(k_{z} R\right) / K_{m}\left(k_{z} R\right)\right)} .
\end{gathered}
$$

in which we have defined

$Q_{0}^{\mathrm{i}}=\frac{\rho_{\mathrm{i}}}{\rho_{0}} \frac{\left(\omega^{2}-\omega_{\mathrm{Ai}}^{2}\right)}{\left(\omega^{2}-\omega_{\mathrm{A} 0}^{2}\right)}, \quad Q_{0}^{\mathrm{e}}=\frac{\rho_{\mathrm{e}}}{\rho_{0}} \frac{\left(\omega^{2}-\omega_{\mathrm{Ae}}^{2}\right)}{\left(\omega^{2}-\omega_{\mathrm{A} 0}^{2}\right)}$.

Equation (11) is the dispersion relation for wave propagation in an incompressible straight magnetic flux tube structure consisting of a core tube and an annulus of radii $a$ and $R$, respectively, embedded in an ambient magnetic environment with straight field lines. This dispersion relation is also attainable from Erdélyi \& Carter (2006) when considering the case when the component of twist $B_{\theta} \equiv 0$. For the limiting case of no core $a \rightarrow 0$ (or equally $a \rightarrow R$ ) we recover a dispersion relation derived by ER83 for a single straight monolithic tube with external Alfvén speed $v_{\mathrm{Ae}}$ and internal Alfvén speed $v_{\mathrm{A} 0}\left(v_{\mathrm{Ai}}\right)$. Mikhalyaev \& Solov'ev (2005) derived the dispersion relation for a core-shell tube in a fully compressible medium. Applying incompressibility $\left(c_{\mathrm{e}}, c_{\mathrm{i}}, c_{0} \rightarrow \infty\right)$ to their equation so that the radial wavenumber becomes

$k_{r}^{2}=\frac{\left(\omega^{2}-v_{\mathrm{A}}^{2} k_{z}^{2}\right)\left(\omega^{2}-c_{\mathrm{s}}^{2} k_{z}^{2}\right)}{\left(c_{s}^{2}+v_{\mathrm{A}}^{2}\right)\left(\omega^{2}-c_{\mathrm{T}}^{2} k_{z}^{2}\right)}=-k_{z}^{2}$

we are able to recover Eq. (11) for the incompressible case. It is of great practical interest to find the difference in phase speeds and hence periods of counterpart waves between those allowed in the annulus geometries and those existing in models developed by ER83. Equation (14) shows the dispersion relation (11) can be re-cast as the sum of the dispersion relation for a straight tube without annulus (ER83) plus an extra term due to a finite annulus.

$\underbrace{Q_{0}^{\mathrm{e}}-\phi_{m R}}_{\text {ER loop }}-\underbrace{\frac{\left(Q_{0}^{\mathrm{e}}-1\right)\left(Q_{0}^{\mathrm{i}}-1\right) \frac{K_{m}^{\prime}\left(k_{z} R\right)}{I_{m}^{\prime}\left(k_{z} R\right)}}{\left(Q_{0}^{\mathrm{i}} \phi_{m a}-1\right) \frac{K_{m}\left(k_{z} a\right)}{I_{m}\left(k_{z} a\right)}}}_{\text {Due to annulus }}=0$,

where

$\phi_{m a}=\frac{I_{m}\left(k_{z} a\right) K_{m}^{\prime}\left(k_{z} a\right)}{K_{m}\left(k_{z} a\right) I_{m}^{\prime}\left(k_{z} a\right)}, \quad \phi_{m R}=\frac{I_{m}\left(k_{z} R\right) K_{m}^{\prime}\left(k_{z} R\right)}{K_{m}\left(k_{z} R\right) I_{m}^{\prime}\left(k_{z} R\right)}$.

\subsection{Analysis and results}

We now consider some specific cases to investigate the behaviour of modes found in this magnetic annulus geometry. It was shown by ER83 that, for the case of incompressibility, there is just one surface mode propagating with a phase speed approximately that of the tube's kink speed defined as $c_{k}=\left(\left(\rho_{\mathrm{i}} v_{\mathrm{Ai}}^{2}+\rho_{\mathrm{e}} v_{\mathrm{Ae}}^{2}\right) /\left(\rho_{\mathrm{i}}+\rho_{\mathrm{e}}\right)\right)^{1 / 2}$. In a single tube, since there is only one surface (at $r=R$ ), this result is intuitive. For the case now under study of a core and annulus we would expect, since there are now two surfaces, i.e. at $r=a$ and $r=R$, to find two separate surface modes occurring with phase speeds approximately corresponding to two kink speeds, respectively,

$c_{k \mathrm{i}}=\sqrt{\frac{\rho_{0} v_{\mathrm{A} 0}^{2}+\rho_{\mathrm{i}} v_{\mathrm{Ai}}^{2}}{\rho_{0}+\rho_{\mathrm{i}}}}, \quad c_{k \mathrm{e}}=\sqrt{\frac{\rho_{0} v_{\mathrm{A} 0}^{2}+\rho_{\mathrm{e}} v_{\mathrm{Ae}}^{2}}{\rho_{0}+\rho_{\mathrm{e}}}}$

at surfaces $r=a$ and $r=R$, being of importance. This is indeed what turns out to be the case.

There are two special cases that we will consider: $v_{\mathrm{Ai}}>$ $v_{\mathrm{A} 0} \gg v_{\mathrm{Ae}}$, modelling a flux tube as having an internal magnetic field much stronger than the external field, applicable perhaps at the interior-atmosphere interface of the Sun, i.e. at photospheric regions, and $v_{\mathrm{Ai}}<v_{\mathrm{A} 0}<v_{\mathrm{Ae}}$, modelling a dense flux tube as an internal density enhancement. We emphasise once more, for kink oscillations the latter is a very good approximation even in low- $\beta$ plasma because of the nature of these practically incompressible oscillations.

\subsubsection{Photospheric loops}

In the first case (with internal Alfvén speeds much greater than external one) we assume that the tube is distinct from its surroundings (and, hence, the modes have different speeds) due to 


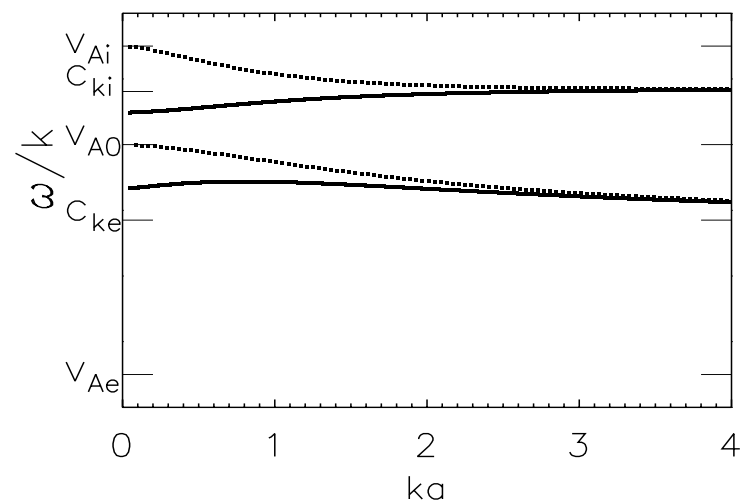

Fig. 2. Dispersion curves for $m=0$ sausage modes (dotted) and for $m=$ 1 kink modes (solid) determined for a magnetic annulus with $a / R=0.8$, giving the phase speed $\omega / k$ as a function of dimensionless wavenumber $k a$ for a typical case mimicking photospheric conditions.

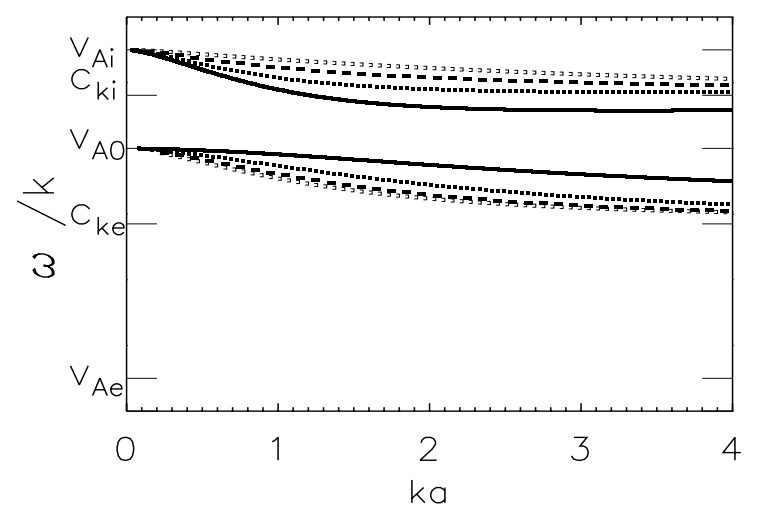

Fig. 3. Sausage modes for the photospheric case for $a / R=0.95$ (solid), 0.8 (dot), 0.6 (dash), 0.5 (open dot).

an enhancement of magnetic field within the core-annulus region, so that $B_{\mathrm{i}}>B_{0} \gg B_{\mathrm{e}}$. We take the densities, for the sake of simplicity, as uniform across the enhancement so that $\rho_{\mathrm{i}}=\rho_{0}=\rho_{\mathrm{e}}$ and hence obtain the condition $v_{\mathrm{Ai}}>v_{\mathrm{A} 0} \gg v_{\mathrm{Ae}}$. Figure 2 shows the dispersion curves for this configuration plotted from Eq. (11). Shown are the two sausage (dotted) and two kink (solid) surface modes. For small values of $k_{z} a$, the two sausage modes approach the speeds $v_{\mathrm{A} 0}$ and $v_{\mathrm{Ai}}$, mimicking the single-surface mode behaviour in ER83 in each case. For $k_{z} a$ large the phase speed of the sausage and kink modes become almost identical - a feature also observed for the single tube model. It is of interest to note that, as found for a single tube, the phase speed of the kink mode at the surface between annulus and external region (with phase speed between $v_{\mathrm{A} 0}$ and $v_{\mathrm{Ae}}$ ) is not monotonic as a function of $k_{z} a$ but has a maximum for $v_{\mathrm{A} 0}>v_{\mathrm{Ae}}$.

The present study investigates what effect the inclusion of an annulus layer has on a monolithic straight magnetic tube. It is of great interest to establish this effect on, e.g. phase speeds or periods supported by the magnetic annulus system.

Figure 3 shows solutions to the dispersion relation Eq. (11) for the sausage modes, and Fig. 4 for the kink modes, for cases with various annulus widths. It is clear from these two plots that the existence and width of an annulus layer has an effect on the phase speeds. A change of annulus width from 5 to 15 percent of the tube width results in a change in period of up to $10 \%$. Note that increasing the thickness of the annulus causes the modes to become less dispersive. This is true except for the

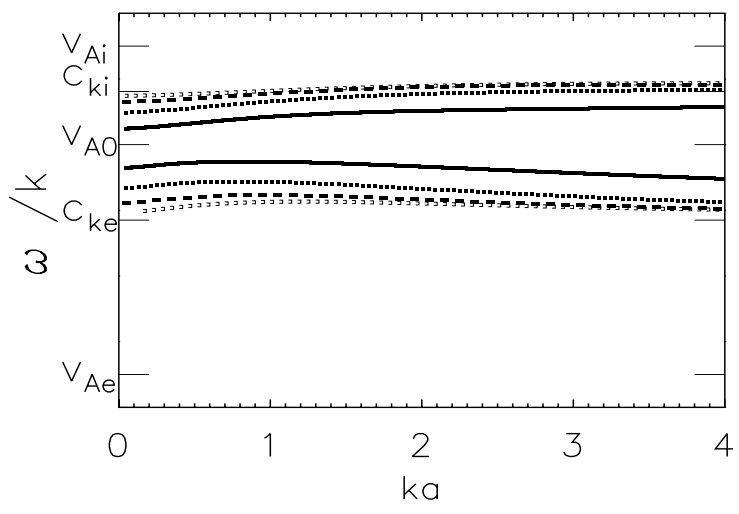

Fig. 4. Kink modes for the photospheric case for $a / R=0.95$ (solid), 0.8 (dot), 0.6 (dash), 0.5 (open dot).

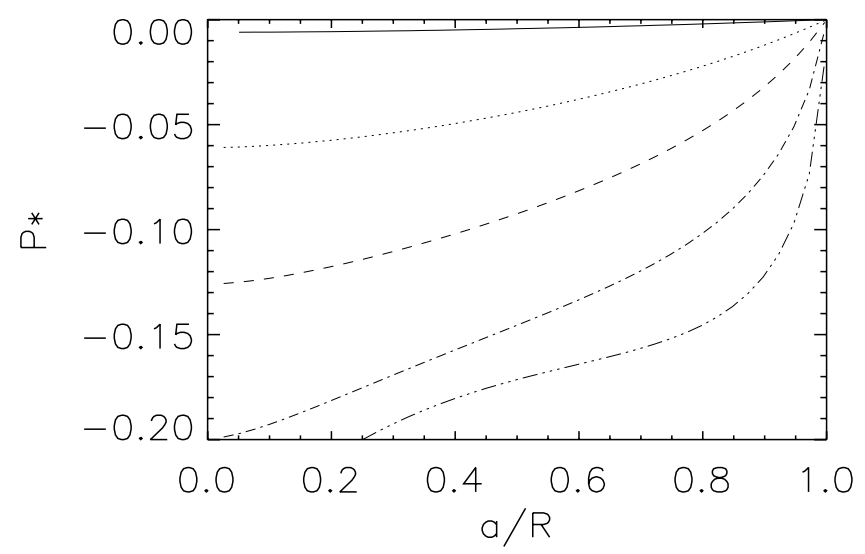

Fig. 5. Plotted is the relative difference, $P^{*}$, between the period, $P_{\mathrm{ER}}$, of the the single tube case and the period, $P$, obtained from Eq. (11) of the sausage modes for varying relative core width $a / R$. $\left(P^{*}=\right.$ $\left(P-P_{\mathrm{ER}}\right) / P_{\mathrm{ER}}$.) Shown are plots for $k a$ values of 0.1 (solid), 0.5 (dot), 1 (dash), 2 (dot-dash) and 4 (dot-dot-dot-dash) for the photospheric case with single tube internal scaling speed $v_{\mathrm{AiER}}=v_{\mathrm{Ai}}$.

external sausage mode. When comparing the phase speeds and periods of the annulus-core geometry to the straight monolithic tube the results are dependent on the relative scaling values chosen for the internal Alfvén speed of the single tube. Two possibilities are immediately apparent; taking the single tube internal speed (denoted as $v_{\text {AiER }}$ ), firstly, corresponding to the core Alfvén speed, $v_{\mathrm{Ai}}$, and secondly corresponding to the annulus Alfvén speed, $v_{\mathrm{A} 0}$.

In the first scenario (when $v_{\mathrm{AiER}}=v_{\mathrm{Ai}}$ ) we find that, as expected, the phase speed of the mode due to the surface between core and annulus approximates the phase speed of this mode for the single monolithic tube. For $v_{\mathrm{AiER}}=v_{\mathrm{Ai}}$, Figs. 5 and 6 are plots of the relative difference, $P^{*}$, between the period, $P$, of the new modes (sausage and kink, respectively) at the inner surface at $r=a$ and the period, $P_{\mathrm{ER}}$, of the modes for the case studied by ER83 (where $\left.P^{*}=\left(P-P_{\mathrm{ER}}\right) / P_{\mathrm{ER}}\right)$ against $a / R$ (the relative core width). Shown are the relative changes for various values of $k_{z} a$. We find that the scaled or relative difference in periods, $P^{*}$, approaches 0 as the width of the annulus region becomes very small $(a / R \rightarrow 1)$. This is an expected result since, physically, as $a / R \rightarrow 1$ we obtain the monolithic geometry. For the sausage modes (Fig. 5) the difference between the modes increases as the wavelength decreases whereas, for the kink modes, there seems to be a minimum, non-zero difference for any particular value of $a / R$ between $k_{z} a=0.5$ and $k_{z} a=2$ (see Fig. 6). 


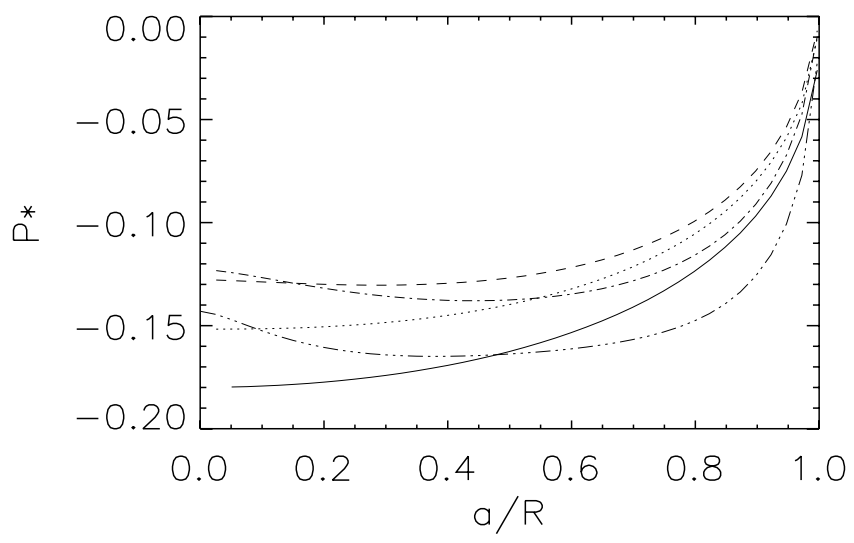

Fig. 6. Same as Fig. 5 but for the kink modes $\left(v_{\mathrm{AiER}}=v_{\mathrm{Ai}}\right)$.

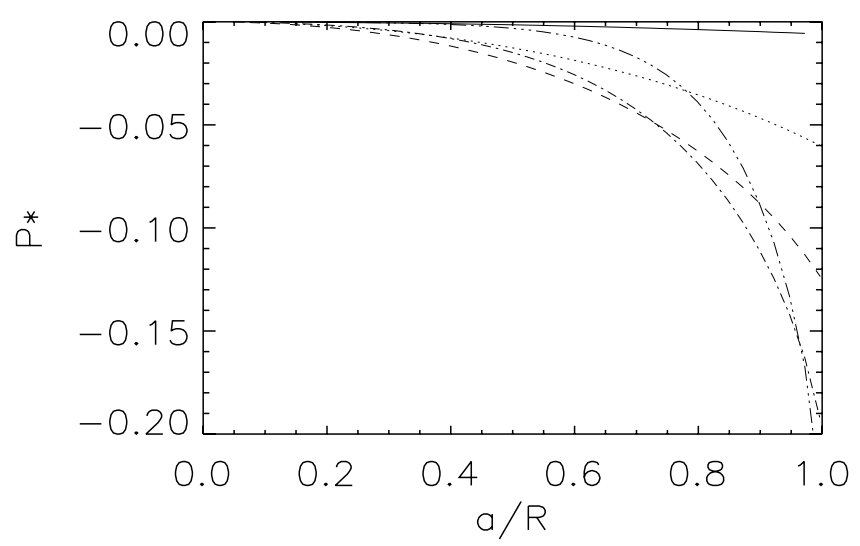

Fig. 7. Same as Fig. 5 for sausage modes in the photospheric case but for scaling with $v_{\mathrm{AiER}}=v_{\mathrm{A} 0}$

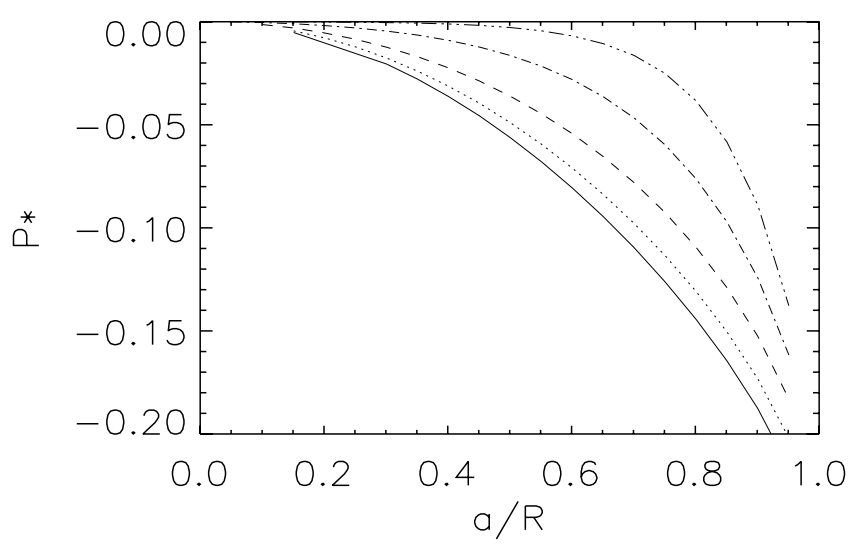

Fig. 8. Same as Fig. 7 but for the kink modes $\left(v_{\mathrm{AiER}}=v_{\mathrm{A} 0}\right)$.

In the case of the second scenario of scaling, when $v_{\mathrm{AiER}}=$ $v_{\mathrm{A} 0}, P^{*}$ is again plotted for various $k_{z} a$ values in Figs. 7 and 8. We find that as $a / R$ decreases (the core region narrows) the difference in periods tend to zero. This is for a comparable reason as before: the tube now approximately becoming the single tube with inner Alfvén speed $v_{\mathrm{AiER}}=v_{\mathrm{A} 0}$. We find that the sausage mode has a maximum difference in periods for a $k_{z} a$ value between 1 and 2 (Fig. 7) whereas the phase speeds of the kink modes of the new geometry and the single tube model become closer as $k_{z} a$ increases (Fig. 8). Finally, and most importantly, we note that all periods are less than their counterparts in a monolithic loop. We conclude that structuring will reduce (increase) periods (frequencies) of the allowed oscillations.

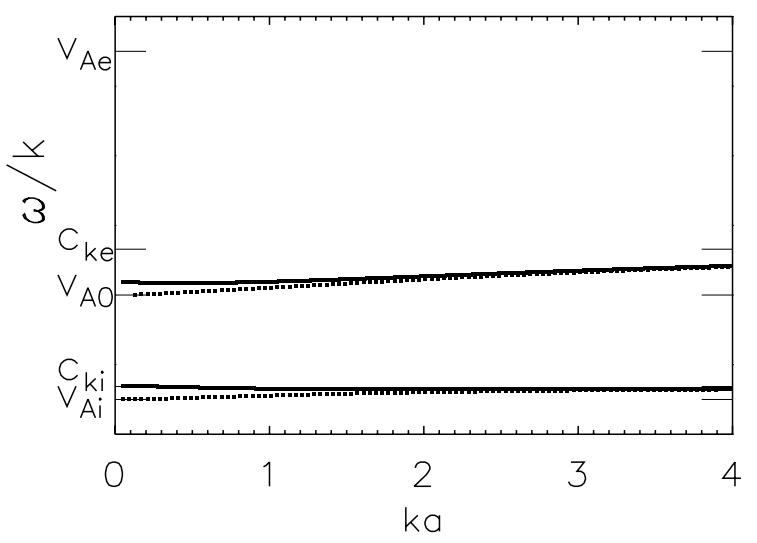

Fig. 9. Dispersion curves of the $m=0$ sausage modes (dotted) and of the $m=1$ kink modes (solid) computed from the dispersion relation with $a / R=0.8$, showing the phase speed $\omega / k$ as a function of the dimensionless wavenumber $k a$ for the case of a tube with a dense core.

\subsubsection{Dense loops}

By considering now the tube as a density enhancement in the core and annulus with (approximate) constant magnetic field strength throughout we have $B_{\mathrm{i}}=B_{0}=B_{\mathrm{e}}, \rho_{\mathrm{i}}>\rho_{0}>\rho_{\mathrm{e}}$ and hence $v_{\mathrm{Ai}}<v_{\mathrm{A} 0}<v_{\mathrm{Ae}}$. The dispersion curves for these characteristic values are shown in Fig. 9 and, again, two distinct surface modes are found for both the sausage and kink modes. In essence these modes have many features similar to the photospheric case. The phase speeds of the sausage modes approach the annulus and core Alfvén speeds $v_{\mathrm{A} 0}$ and $v_{\mathrm{Ai}}$ as $k_{z} a \rightarrow 0$ and the sausage and kink modes have the approximate same phase speed for large $k_{z} a$. The two modes (sausage and kink) at the inner surface $r=a$ (outer surface $r=R$ ) are confined to the interval $\left[v_{\mathrm{Ai}}, c_{k \mathrm{i}}\right]\left(\left[v_{\mathrm{A} 0}, c_{k \mathrm{e}}\right]\right)$, a similar result to that found for the photospheric case. The kink mode at the external boundary $r=R$ is not monotonic but has a minimum phase speed, although it is now less distinct than in the photospheric case.

Let us now investigate what effect a finite annulus will have on the modes' phase speeds and periods. Figures 10 and 11, which plot the sausage and kink modes for various annulus widths from $a / R=0.4$ to 0.95 , clearly show that the existence and width of the annulus layer can be significant once more. The effects of varying $a / R$ (the core width) on the obtained periods are shown in Figs. $12-15$ which plot $a / R$ against $P^{*}\left(=\left(P-P_{\mathrm{ER}}\right) / P_{\mathrm{ER}}\right)$.

Figures 12 and 13 are plots of the sausage and kink modes, respectively, for an internal single tube speed, $v_{\text {AiER }}$, equal to the core speed, $v_{\mathrm{Ai}}$, in the core-annulus model. In both plots the relative period difference, $P^{*}$, tends to zero as $a / R \rightarrow 1$ (i.e. as we approach the single tube model with internal speed $v_{\mathrm{Ai}}$ ). The difference in period for the sausage mode (Fig. 12) increases as $k_{z} a$ does with an apparent maximum for a $k_{z} a$ value between 1 and 4 . The relative period difference for the kink mode (Fig. 13), apart from tending to zero as $a / R \rightarrow 1$, has a less obvious trend. This is due to the modes being approximately equal in the two cases and crossing at different values of $k_{z} a$.

In the case when the scaling is taken as $v_{\mathrm{AiER}}=v_{\mathrm{A} 0}$ we find that the two periods from the two geometries approach as $a / R$ tends to zero. Figures 14 and 15 show the sausage and kink modes for this case. Again, the sausage mode has a maximum difference in period for a $k_{z} a$ value between 1.5 and 2 (Fig. 14) whereas the kink mode period differs more from the straight tube period as $k_{z} a$ decreases (Fig. 15). Most importantly note that, for 


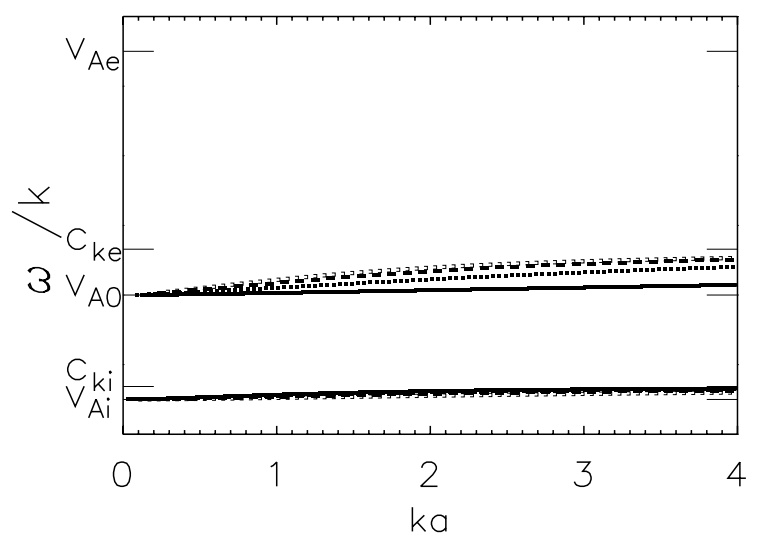

Fig. 10. Sausage modes for the case of a dense tube determined for $a / R=0.95$ (solid), 0.8 (dot), 0.6 (dash), 0.5 (open dot).

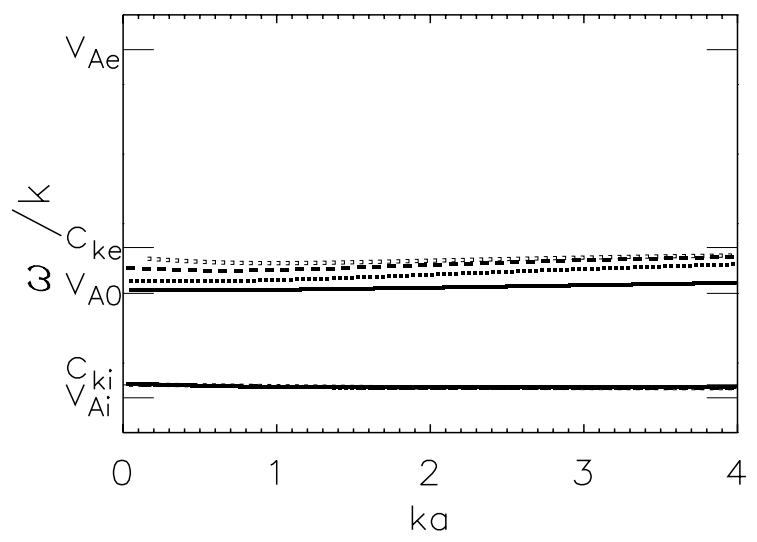

Fig. 11. Kink modes for the case of a dense tube determined for $a / R=$ 0.95 (solid), 0.8 (dot), 0.6 (dash), 0.5 (open dot).

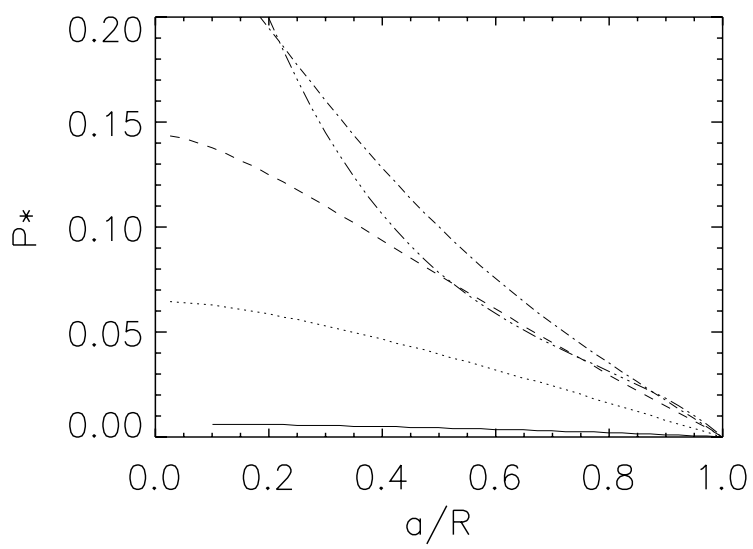

Fig. 12. Plotted is the relative difference, $P^{*}$, between the period, $P_{\mathrm{ER}}$, of the the single monolithic tube case and the period, $P$, obtained from the dispersion relation of the sausage modes for a range of relative core width $a / R$ for the dense tube case with single tube internal scaling speed $v_{\mathrm{AiER}}=v_{\mathrm{Ai}}\left(P^{*}=\left(P-P_{\mathrm{ER}}\right) / P_{\mathrm{ER}}\right)$. Shown are plots for $k a$ values of 0.1 (solid), 0.5 (dot), 1 (dash), 2 (dot-dash) and 4 (dot-dot-dot-dash).

the majority of cases, periods $P$ are greater than the periods $P_{\mathrm{ER}}$ of the counterpart monolithic flux tube.

\subsection{Asymptotic expansion}

Finally, it is of interest to study the behaviour of the dispersion relation, Eq. (11), describing a flux tube embedded in another

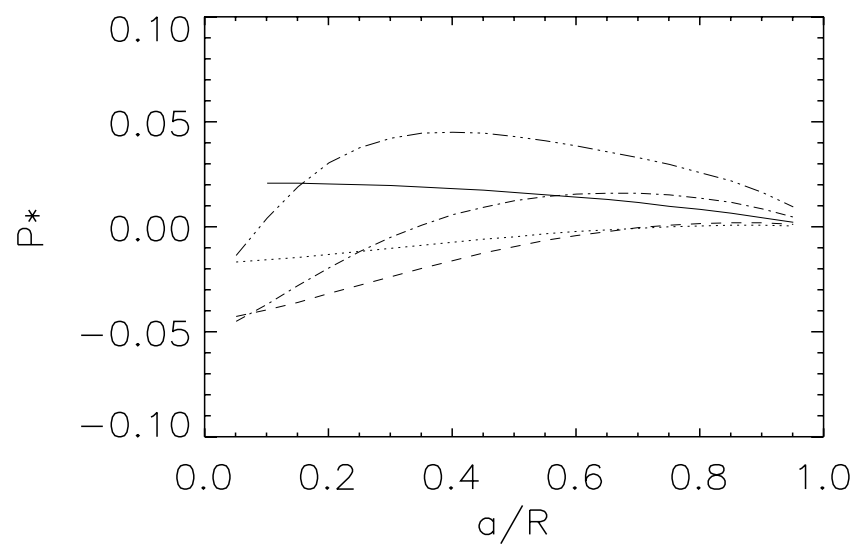

Fig. 13. Same as Fig. 12 but for the kink modes.

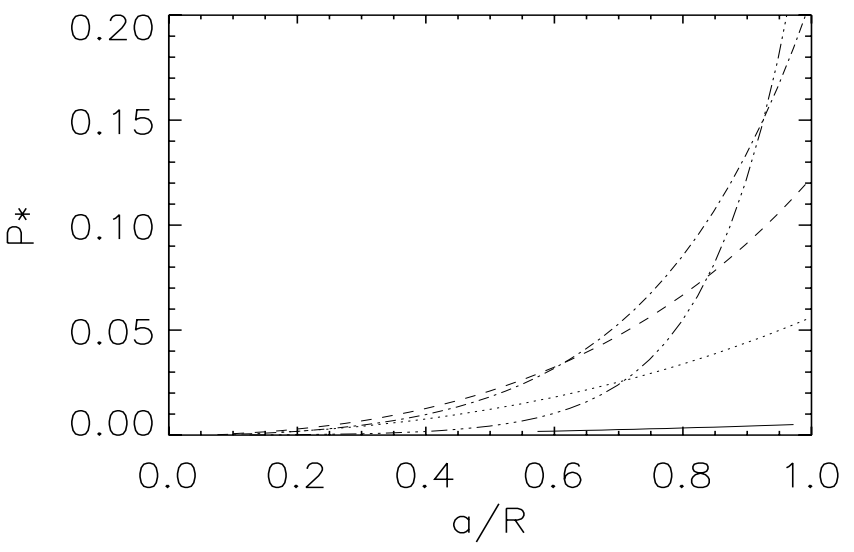

Fig. 14. Same as Fig. 12 but for sausage modes with $v_{\mathrm{AiER}}=v_{\mathrm{A} 0}$.

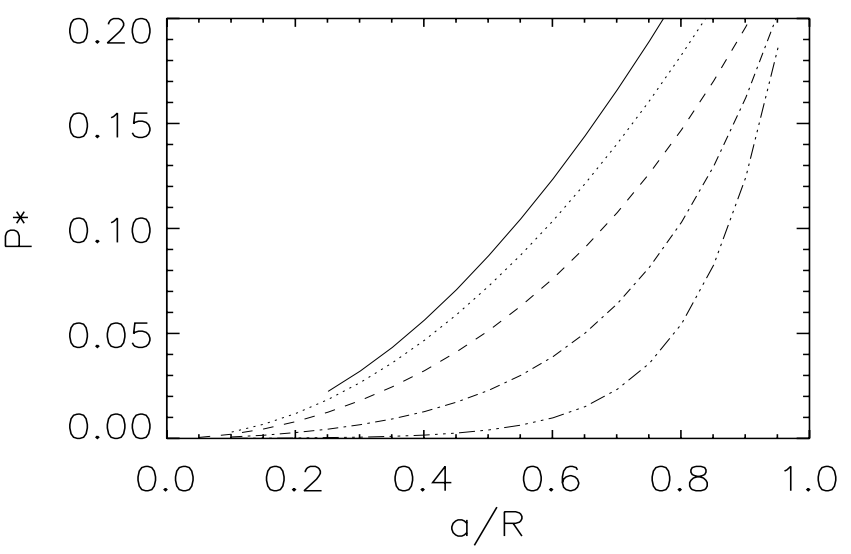

Fig. 15. Same as Fig. 14 but for the kink modes.

tube in the extremes of large and small $k_{z} a$. We introduce the notation $x=k_{z} a, y=k_{z} R$ for the following analysis.

\subsubsection{Long wavelength $(x<y \ll 1)$}

Sausage modes. We first consider the long wavelength approximation for the sausage modes, $m=0$. Letting $k_{z} a<k_{z} R \ll 1$ and, as a consequence, expanding the Bessel functions $K_{0}^{\prime}, I_{0}^{\prime}$ and $I_{0}$ in Eq. (11) we obtain

$$
\frac{Q_{0}^{\mathrm{i}}\left(\frac{-1}{x}\right)-\frac{x}{2} K_{0}(x)}{\left(Q_{0}^{\mathrm{i}}-1\right) \frac{x}{2}}=\frac{\left(Q_{0}^{\mathrm{e}}-1\right) \frac{-1}{y}}{Q_{0}^{\mathrm{e}}\left(\frac{y}{2}\right)-\frac{1}{y K_{0}(y)}} .
$$


B. K. Carter and R. Erdélyi: Sausage and kink oscillations in incompressible annular magnetic cylinders

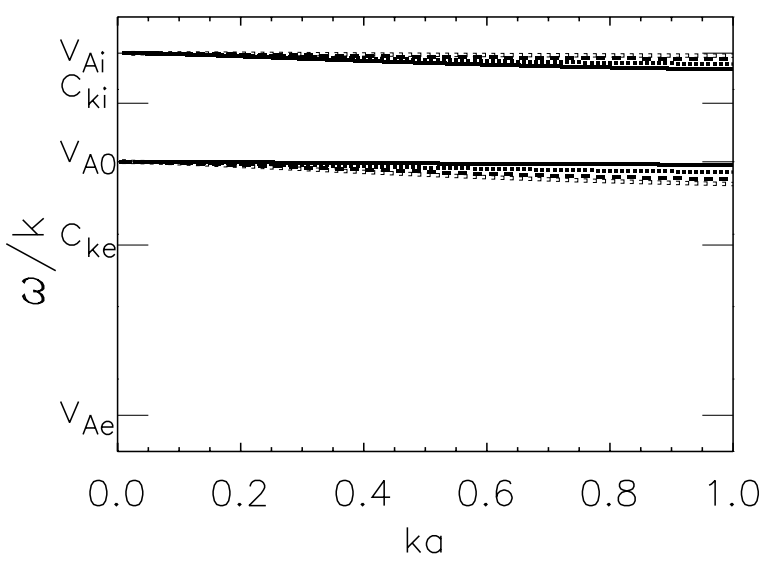

Fig. 16. Phase speed as a function of the dimensionless wavenumber in the limit of long wavelength approximation obtained by solving the approximate dispersion relation Eq. (17) for the sausage mode for typical photospheric values. Shown are curves for $a / R=0.95$ (solid), 0.8 (dot), 0.6 (dash), 0.5 (open dot).

After some algebra, eliminating powers of $k_{z} a$ greater than $\left(k_{z} a\right)^{2}$ (i.e. greater than $x^{2}$ ), we find

$\frac{1+Q_{0}^{\mathrm{e}} \frac{y^{2}}{2} K_{0}(y)}{K_{0}(y)}=\frac{\left(Q_{0}^{\mathrm{i}}-1\right)\left(Q_{0}^{\mathrm{e}}-1\right) \frac{x^{2}}{2}}{Q_{0}^{\mathrm{i}}}$,

Figs. 16, 17 are plots of Eq. (17) of the sausage modes for the photospheric and dense tube cases, respectively.

Kink Modes. Let us now consider the kink mode, $m=1$. The dispersion relation (11) in the limit of $k_{z} a<k_{z} R \ll 1$ reduces to

$\frac{\left(Q_{0}^{\mathrm{i}}-1\right)\left(Q_{0}^{\mathrm{e}}-1\right)}{\left(Q_{0}^{\mathrm{i}}+1\right)\left(Q_{0}^{\mathrm{e}}+1\right)}(a / R)^{2}-1=0$

which is independent of the wavenumber $k_{z}$. Equation (18) is quadratic in $\omega^{2}$ and can be solved to find $\omega$ in terms of the Alfvén speeds $v_{\mathrm{Ai}}, v_{\mathrm{A} 0}$ and $v_{\mathrm{Ae}}$ and the densities $\rho_{\mathrm{i}}, \rho_{0}$ and $\rho_{\mathrm{e}}$ :

$$
\begin{aligned}
\left(\omega / k_{z}\right)^{2} & =\left(c_{k \mathrm{i}}^{2}+c_{k \mathrm{e}}^{2}\right) \\
& \pm \sqrt{\left(\frac{c_{k \mathrm{i}}^{2}-c_{k \mathrm{e}}^{2}}{2}\right)^{2}+(a / R)^{2}\left(v_{\mathrm{A} 0}^{2}-v_{\mathrm{Ai}}^{2}\right)\left(v_{\mathrm{A} 0}^{2}-v_{\mathrm{Ae}}^{2}\right)}
\end{aligned}
$$

for $\rho_{\mathrm{i}}=\rho_{0}=\rho_{\mathrm{e}}$ and

$$
\begin{aligned}
\left(\omega / k_{z}\right)^{2} & =\frac{\mathcal{R}\left(c_{1}^{2}+c_{2}^{2}\right)-\left(c_{k \mathrm{i}}^{2}+c_{k \mathrm{e}}^{2}\right)}{2(\mathcal{R}-1)} \\
& \pm \sqrt{\frac{\left(\mathcal{R}\left(c_{2}^{2}-c_{1}^{2}\right)-\left(c_{k \mathrm{e}}^{2}-c_{k \mathrm{i}}^{2}\right)^{2}+4 \mathcal{R}\left(c_{k \mathrm{e}}^{2}-c_{2}^{2}\right)\left(c_{k \mathrm{i}}^{2}-c_{1}^{2}\right)\right.}{4(\mathcal{R}-1)^{2}}}
\end{aligned}
$$

for $\rho_{\mathrm{i}} \neq \rho_{0} \neq \rho_{\mathrm{e}}$ in which

$\mathcal{R}=\frac{\left(\rho_{0}-\rho_{\mathrm{i}}\right)\left(\rho_{0}-\rho_{\mathrm{e}}\right)(a / R)^{2}}{\left(\rho_{0}+\rho_{\mathrm{i}}\right)\left(\rho_{0}+\rho_{\mathrm{e}}\right)}$,

$c_{1}^{2}=\frac{\rho_{0} v_{\mathrm{A} 0}^{2}-\rho_{\mathrm{i}} v_{\mathrm{Ai}}^{2}}{\rho_{0}-\rho_{\mathrm{i}}}$,

$c_{2}^{2}=\frac{\rho_{0} v_{\mathrm{A} 0}^{2}-\rho_{\mathrm{e}} v_{\mathrm{Ae}}^{2}}{\rho_{0}-\rho_{\mathrm{e}}}$.

Figure 18, for $v_{\mathrm{Ai}}>v_{\mathrm{A} 0} \gg v_{\mathrm{Ae}}, \rho_{\mathrm{i}}=\rho_{0}=\rho_{\mathrm{e}}$, and Fig. 19, for $v_{\mathrm{Ai}}<v_{\mathrm{A} 0}<v_{\mathrm{Ae}}$, show the relationship between $a / R$ and phase speed $\omega$ for kink oscillations in an incompressible shell model in the long wavelength approximation described in Eq. (18).

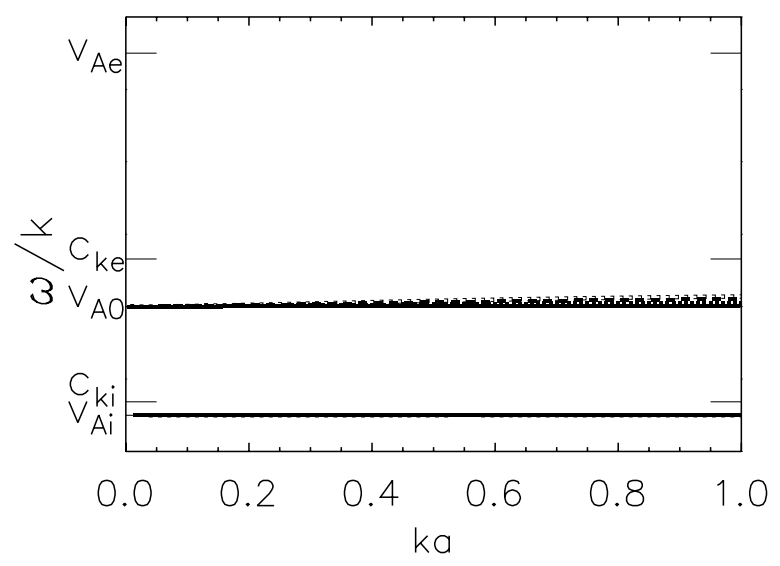

Fig. 17. Same as Fig. 16 but for a dense tube $\left(v_{\mathrm{Ai}}<v_{\mathrm{A} 0}<v_{\mathrm{Ae}}\right)$.

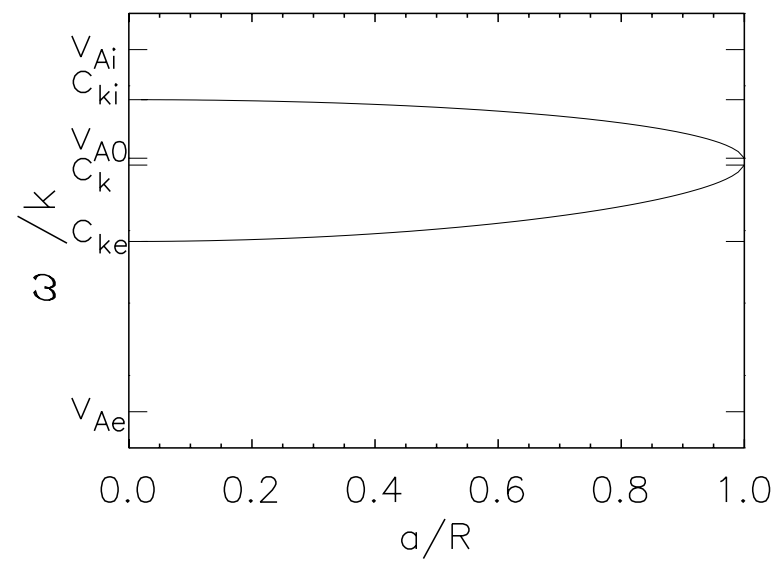

Fig. 18. Phase speed as a function of $a / R$ (the ratio of core and annulus radii) in the limit of long wavelength approximation Eq. (19a) for the kink mode for $v_{\mathrm{Ai}}>v_{\mathrm{A} 0}>v_{\mathrm{Ae}}, \rho_{\mathrm{i}}=\rho_{0}=\rho_{\mathrm{e}}$. The phase speed for these curves is independent of wavenumber $k_{z}$.

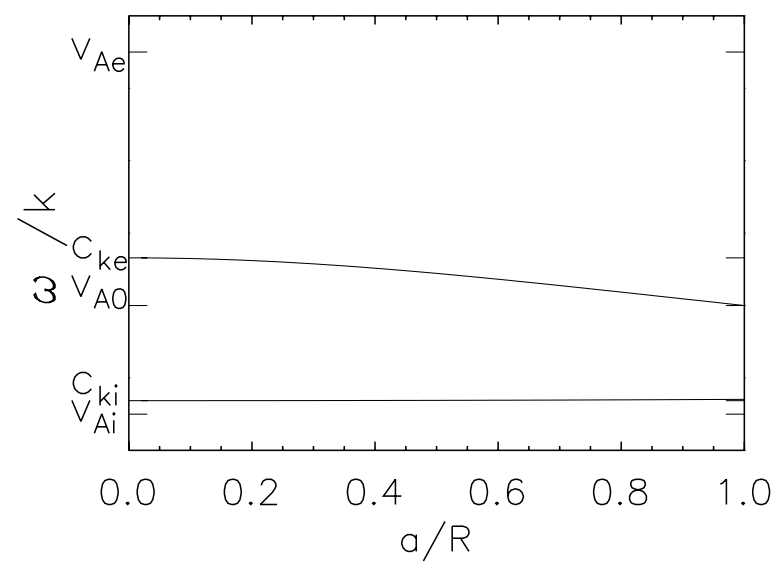

Fig. 19. Same as Fig. 18 but from Eq. (19b) for $v_{\mathrm{Ai}}<v_{\mathrm{A} 0}<v_{\mathrm{Ae}}$.

\subsubsection{Short wavelength, $k_{z} R>k_{z} a \gg 1$}

In the case of large $z$ it is useful to note that

$$
\begin{aligned}
& \frac{K_{0}^{\prime}(z)}{K_{0}(z)} \approx-1-\frac{1}{2 z}, \quad \frac{K_{1}^{\prime}(z)}{K_{1}(z)} \approx-1-\frac{1}{2 z}, \\
& \frac{I_{0}^{\prime}(z)}{I_{0}(z)} \approx 1-\frac{1}{2 z}, \quad \frac{I_{1}^{\prime}(z)}{I_{1}(z)} \approx 1-\frac{1}{2 z} .
\end{aligned}
$$




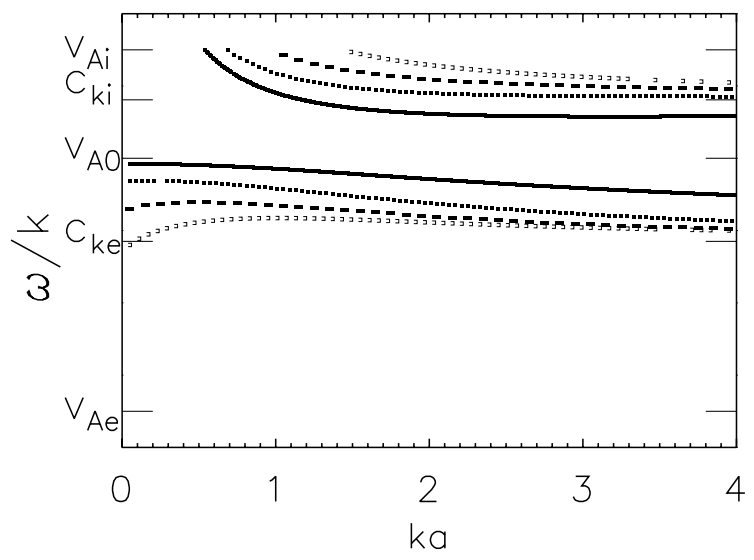

Fig. 20. Short wavelength plot of Eq. (22) for $v_{\mathrm{Ai}}>v_{\mathrm{A} 0}>v_{\mathrm{Ae}}$. Again shown are curves for $a / R=0.95$ (solid), 0.8 (dot), 0.6 (dash), 0.5 (open dot).

So that, in the short wavelength approximation, the dispersion relation (and hence the phase speed and existence of modes) are not dependant on the value of the azimuthal wavenumber $m<2$. This is indicative of the short wavelength tendencies in the plots, Figs. 2 and 9, of the full dispersion relation.

We are, for the short wavelength approximation, now able to reduce the general dispersion relation (11) to

$$
\begin{aligned}
\mathrm{e}^{2 k_{z}(R-a)}= & \frac{\left(Q_{0}^{\mathrm{i}}-1\right)\left(Q_{0}^{\mathrm{e}}-1\right)}{\left(Q_{0}^{\mathrm{i}}+1\right)\left(Q_{0}^{\mathrm{e}}+1\right)} \\
& \times\left\{1-\frac{Q_{0}^{\mathrm{i}}}{\left(Q_{0}^{\mathrm{i}}+1\right) k_{z} a}+\frac{Q_{0}^{\mathrm{e}}}{\left(Q_{0}^{\mathrm{e}}+1\right) k_{z} R}\right\},
\end{aligned}
$$

which holds for both $m=0$ and $m=1$. Equation (22) is solved numerically and the solution for the dispersion relation is plotted in Figs. 20 and 21. Further, for $k_{z}(R-a) \ll 1$ (narrow annulus), we find that

$\mathrm{e}^{2 k_{z}(R-a)} \approx 1$

and so Eq. (22) reduces to

$$
1 \approx \frac{\left(Q_{0}^{\mathrm{i}}-1\right)\left(Q_{0}^{\mathrm{e}}-1\right)}{\left(Q_{0}^{\mathrm{i}}+1\right)\left(Q_{0}^{\mathrm{e}}+1\right)}
$$

which yields the analytic solutions

$$
\left(\frac{\omega}{k}\right)^{2}=\frac{\rho_{\mathrm{i}} v_{\mathrm{Ai}}^{2}+\rho_{\mathrm{e}} v_{\mathrm{Ae}}^{2}}{\rho_{\mathrm{i}}+\rho_{\mathrm{e}}}=c_{k}^{2}
$$

and

$$
\left(\frac{\omega}{k}\right)^{2}=\left(\frac{\omega_{\mathrm{A} 0}}{k}\right)^{2}=v_{\mathrm{A} 0}^{2}
$$

where $c_{k}$ is the kink speed of the single tube case with internal speed $v_{\mathrm{Ai}}$ and external speed $v_{\mathrm{Ae}}$ and where $v_{\mathrm{A} 0}$ is the Alfvén speed in the annulus region. However, the approximation $k_{z}(R-$ a) $\ll 1$ coupled with the short wavelength limit implies that the value of $a / R$, and hence also the annulus width, become zero to first order. Physically this means that we are only left with the single tube kink speed $c_{k}$ as a realistic solution.

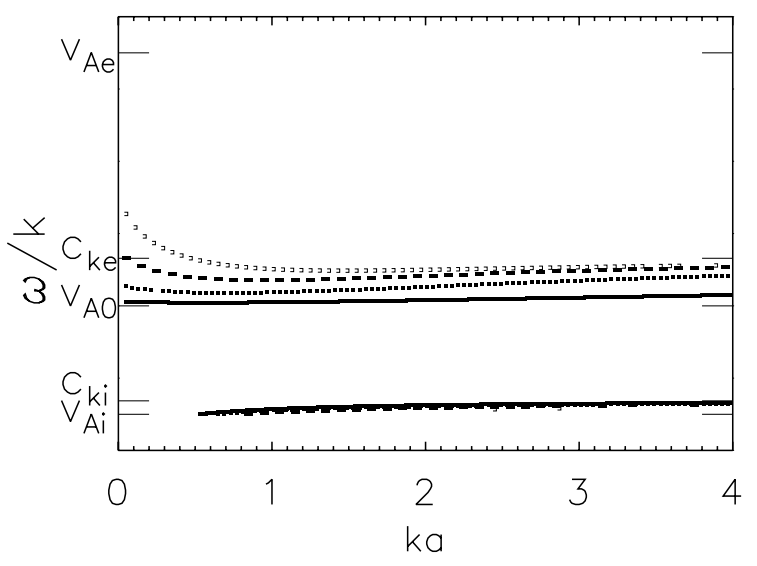

Fig. 21. Same as Fig. 20 but for $v_{\mathrm{Ai}}<v_{\mathrm{A} 0}<v_{\mathrm{Ae}}$.

\section{Concluding remarks}

In this work the oscillations of a magnetic flux tube consisting of a core, annulus and ambient external field embedded in an incompressible plasma are investigated. For simplicity both stratification effects and compressibility are neglected with emphasis being on the effect of the annulus layer on the phase speeds of existing modes. The obtained surface modes of this annulus-core configuration are compared with oscillations of a single straight monolithic tube (as in Edwin \& Roberts 1983) and the change in periods are studied.

The general dispersion relation is found for this model, writable as the dispersion relation for the straight tube plus terms due to the annulus. Due to the three distinct regions there are now three characteristic Alfvén speeds in the magnetic annulus geometry, $v_{\mathrm{Ai}}, v_{\mathrm{A} 0}$ and $v_{\mathrm{Ae}}$ and there are two kink speeds $c_{k \mathrm{i}}$ and $c_{k \mathrm{e}}$ due to the discontinuities at the two surfaces at $r=a$ and $r=R$. The obtained dispersion relation is investigated numerically for two specific cases firstly considering the flux tube as a magnetic field enhancement $\left(v_{\mathrm{Ai}}>v_{\mathrm{A} 0} \gg v_{\mathrm{Ae}}, \rho_{\mathrm{i}}=\rho_{0}=\rho_{\mathrm{e}}\right)$ and secondly as a density enhancement $\left(\rho_{\mathrm{i}}>\rho_{0}>\rho_{\mathrm{e}}, v_{\mathrm{Ai}}<v_{\mathrm{A} 0}<v_{\mathrm{Ae}}\right)$.

There is just one surface mode that occurs in the monolithic tube case but there are now two surface modes arising for both the sausage and kink modes for the annulus-core model. The two sausage modes approach the Alfvén speeds of the core and the annulus at long wavelengths. This result is confirmed by the asymptotic analysis carried out in Sect. 2.5. The two kink modes approach the kink speeds $c_{k \mathrm{i}}$ and $c_{k \mathrm{e}}$ for shorter wavelengths and for larger annulus widths.

Next the effect the annulus layer has on the periods of the modes is investigated, specifically as a comparison to the single straight tube model. It was found that the change in periods of existing modes was significant (up to $20 \%$ in some cases).

For the sausage modes there was a greater difference in period for shorter wavelengths in all cases (see e.g. Figs. 5, 7, 12, 14). In contrast, the kink mode was effected most by the annulus width for longer wavelengths (see e.g. Figs. 6, 8, 13, 15). Most importantly, it was discovered that the additional structuring of the annulus layer caused the periods to be increased in the case of a dense flux tube but decreased for a flux tube modelled as a magnetic field enhancement.

The existence and width of an annulus layer, when included in a simple tube model, has been shown to have an important, and under certain conditions non-negligible, effect on both the existence of and the periods of modes occurring. The addition of a shell, or annulus, layer resulted in the existence of two surface modes where the monolithic tube had solely one surface mode 
for the incompressible case. These surface modes (two sausage and two kink) have periods that are significantly dependent on the width of this layer.

The analysis and results are applicable for the entire solar atmosphere for kink modes only, while for sausage modes the incompressibility condition restricts applicability to high- $\beta$ regions.

Acknowledgements. The authors thank M. Ruderman and N. Venkov for a number of useful discussions. RE acknowledges M. Kéray for patient encouragement. The authors are also grateful to NSF, Hungary (OTKA, Ref. No. TO43741) and to The University of Sheffield (White Rose Consortium) for the financial support they received.

\section{References}

Aschwanden, M. J. 2003, Review of coronal oscillations, in Turbulence, Waves and Instabilities in the Solar Plasma, ed. R. Erdélyi et al., NATO Sci Ser., 124, 215

Ashwanden, M. J. 2004, Physics of the Solar Corona (Chichester, UK: Praxis Publishing Ltd.)

Aschwanden, M. J., Fletcher, L., Schrijver, C. J., et al. 1999, ApJ, 520, 880 Banerjee, D., Erdélyi, R., O’Shea, E., \& Oliver, R. 2007, Sol. Phys., in press Bennett, K., Roberts, B., \& Narain, U. 1999, Sol. Phys., 185, 41

Bogdan, T. J. 2000, Sol. Phys., 192, 373
Bogdan, T. J., \& Judge, P. G. 2006, Phil. Trans. Roy. Soc. A, 364, 313

Christopoulou, E. B., Georgakilas, A. A., \& Koutchmy, S. 2000, A\&A, 354, 305 Christopoulou, E. B., Georgakilas, A. A., \& Koutchmy, S. 2001, A\&A, 375, 617 De Pontieu, B., Tarbell, T., \& Erdélyi, R. 2003, ApJ, 590, 502

De Pontieu, B., Erdélyi, R., \& James, S. P. 2004, Nature, 430, 536

De Pontieu, B., Erdélyi, R., \& De Moortel, I. 2005, ApJ, 624, 61

Edwin, P. M., \& Roberts, B. 1983, Sol. Phys., 88, 179

Erdélyi, R. 2006, Phil. Trans. Roy. Soc. A, 364, 351

Erdélyi, R., \& Carter, B. K. 2006, A\&A, 455, 361

Erdélyi, R., \& Verth, G. 2007, A\&A, 462, 743

Georgakilas, A. A., Christopoulou, E. B., \& Koutchmy, S. 2000, A\&A, 363, 306

Georgakilas, A. A., Muglach, K., \& Christopoulou, E. B. 2002, ApJ, 576, 561

Kobanov, N. I. 2000, Astron. Rep., 44, 202

Kukhianidze, V., Zaqarashvili, T. V., \& Khutsishvili, E. 2003, A\&A, 449, 35

Mikhalyaev, B. B., \& Solov'ev, A. A. 2005, Sol. Phys., 227, 249

Nakariakov, V. M., \& Roberts, B. 2003, in Turbulence, Waves and Instabilities in the Solar Plasma, ed. R. Erdélyi et al., NATO Sci Ser., 124, 167

Nakariakov, V. M., \& Verwichte, E. 2005, LRSP, 2, 3

Nakariakov, V. M., Ofman, L., Deluca, E. E., et al. 1999, Science, 285, 862

Roberts, B. 2000, Sol. Phys., 193, 139

Roberts, B., Edwin, P. M., \& Benz, A. O. 1984, ApJ, 279, 857

Robbrecht, E., Verwichte, E., Berghmans, D., et al. 2001, A\&A, 370, 591

Ryutov, D. A., \& Ryutova, M. P. 1976, Sov. Phys., 43-3, 491

Verth, G., Van Doorsselaere, T., Erdélyi, R., et al. 2007, A\&A, submitted

Wang, T. J. 2004, in Waves, oscillations and small scale transient events in the solar atmosphere: A joint view of SOHO and TRACE, ed. R. Erdélyi et al., ESA SP-547, 417 